\title{
Internal dynamic partial reconfiguration for real time signal processing on FPGA
}

\author{
Sheetal U. Bhandari ${ }^{1}$, Shaila Subbaraman ${ }^{2}$, Shashank Pujari ${ }^{3}$ and Rashmi Mahajan ${ }^{4}$ \\ $1,3,4$ Dept. of Microelectronics and VLSI Design, International Institute of Information Technology, Pune-411057 India \\ ${ }^{2}$ Department of Electronics Engineering, Walchand College of Engg., Sangli 416415 India \\ 1sheetalubhandari@gmail.com, 2shailasubbaraman@yahoo.co.in, 3pujarishashank@gmail.com, \\ ${ }^{4}$ mahajanrashmi@ymail.com
}

\begin{abstract}
Few FPGAs support creation of partially reconfigurable systems when compared to traditional systems based on total reconfiguration. This allows dynamic change of the functionalities hosted on the device when needed and while the rest of the system continues its working. Runtime partial reconfiguration of FPGA is an attractive feature which offers countless benefits across multiple industries. Xilinx has supported partial reconfiguration for many generations of devices. This can be taken advantage of substituting inactive parts of hardware systems and to adapt the complete chip a different requirement of an application. This paper describes an innovative implementation for real time audio and video processing using run time internal partial reconfiguration. System is implemented on Virtex-4 FPGA. Internal reconfiguration is handled using internal configuration access port (ICAP) driven by soft processor core. The considerable savings in device resources, bit stream size and configuration time is observed and tabulated in this paper.
\end{abstract}

Keywords: Reconfigurable computing; partial reconfiguration; run time reconfiguration; internal reconfiguration.

\section{Introduction}

Nowadays, pervasive systems take more and more space in our life as an embedded systems which automatically adapts to change in their environment and act on the base of user needs. Security aspects, seamless communication and self configuration are the key challenges raised by pervasive systems. Flexibility in hardware platform is important to achieve high performance. Run time partial reconfiguration $(P R)$ is an exact candidate for this. Run time PR is a recent method in reconfigurable computing to update selectively the circuitry of a programmable board, while still being active (Fig.1). This allows changing a group of logic very quickly when the application needs it. As it is not possible to plan at design time the evolution of the functions used by pervasive systems (for e.g cryptography, signal processing \& communication protocols), the system must be able to update itself in order to adapt to its environment (Lagger, 2006).

The pervasive systems in consumer electronic domain provide day to day larger amount of functionalities like video-audio processing, communications, entertainment etc. At the same time these functionalities demand more complex support: operating systems, secured communication etc. Guaranteeing high performance is not possible when the processing is fully performed by software. A common approach to improve performance is to include specialize hardwired coprocessor. However, given their static architecture, these systems lack flexibility and having specialized coprocessor for each

Research article (CIndian Society for Education and Environment (iSee)

task is not feasible, due to the amount of logic required and the possible incompatibility of upgraded version of the algorithms. The system must be able to update its own hardware platform in order to adapt to an environment in autonomous way. Dynamic reconfiguration can deal with this problem by modifying their function without altering the rest of the system with minimum resources.

In this paper we present innovative real time audio, video signal processing using Internal PR feature of FPGA. This approach shows considerable savings in area and reconfiguration time too. In the following section, the concept of internal run time partial reconfiguration is discussed; followed by briefing of processing real time audio and video signal. Hardware platform, processor and operating system are also described before presenting results and discussions.

Run time internal partial reconfiguration

With rising gate densities and increased power of FPGA, co-existence of processor and digital logic components is possible on single device. This provides flexibility of combining software and hardware based control in one chip. One more important feature is being added to FPGA to further enhance the performance with minimum resources is partial reconfiguration. This feature

Fig. 1. The concept of partial reconfiguration

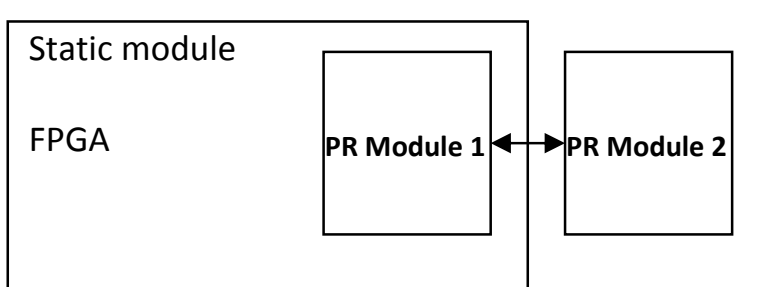

"Real time signal processing" http://www.indjst.org is provided in few FPGAs from Xilinx like Virtex II, Virtex IV etc (Koo, 2005). On the fly PR provides a way to modify the implemented logic in FPGA when the device is on. More clearly $P R$ allows reconfiguring selected areas of a FPGA when other part of FPGA is still

Sheetal et al. Indian J.Sci.Technol. 
working. There are different models of reconfiguration classified on the basis of who performs it when it happens and on which level of granularity it is happening. On the fly changes can be done to single element like DCM, LUT etc or a module consisting of these components. Xilinx has proposed two flows of PR for these granularity levels, difference based and module based PR respectively (XAPP290, 2004). Configuration generation can be done in complete static way, at design time, determining all possible configurations of the systems. Each module must be synthesized and all possible connections between modules and the rest of the system must be considered. Other possibilities are runtime placement of pre-synthesized modules, which requires dynamic routing of interconnection signals or complete dynamic modules generation. On the fly reconfiguration can be handled by external entity like PC or by FPGA itself. Handling this reconfiguration internally by FPGA provides autonomy. The subset of select-map core is an internal access configuration port (ICAP), which is used for writing on-the-fly partial configuration bit streams in the FPGA (Xapp138, 2000).

Audio video signal processing

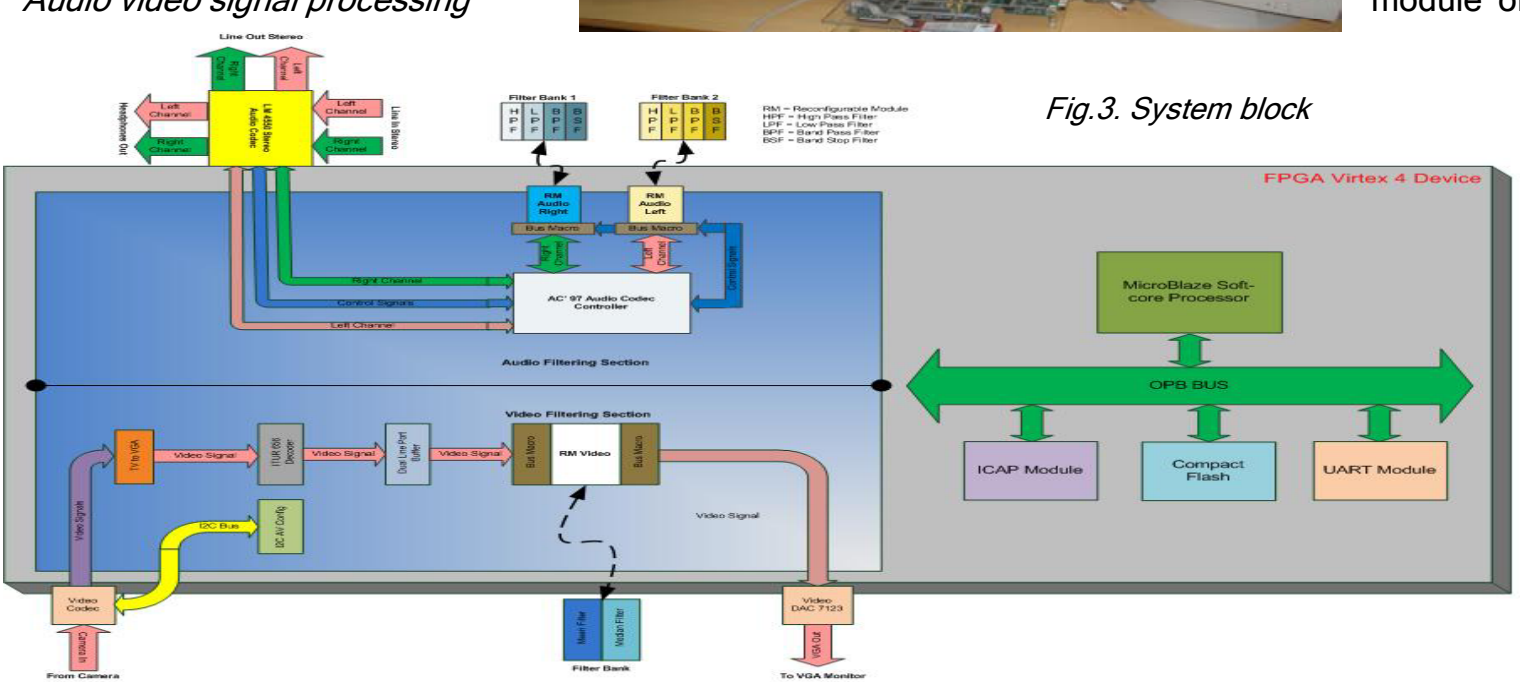
neighborhood. Fig.2. Set up

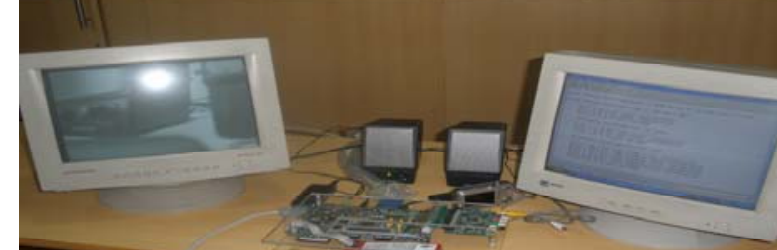

based on following four modules. (1) Real time calculation of Intensity of each pixel. (2) Storage of 5 lines of RGB pixels. (3) Calculation of average intensity of center pixel w.r.t. surrounding $5 \times 5$ pixels' intensity (4) Regeneration of R, G, B of center pixel as function of this new average intensity. In median filtering the input pixel is replaced by the median of the pixels contained in the

The algorithm for median filtering requires sorting the pixel gray values in the neighborhood in increasing or decreasing order and picking up the median of the array (Oppenheim \& Schafer, 2000; Chanda et al., 2003). The methodology of median filtering is based on following four modules. (1) Real time calculation of Intensity of each pixel. (2) Storage of 5 lines of RGB pixels. (3) Finding out the median intensity of center pixel w.r.t. Surrounding $5 \mathrm{x}$ 5 pixels' intensity. (4) Regeneration of R, G, B of center pixel as function of this new average intensity. As any one filter would require at any time, instead of implementing two filters, third step of above is implemented as dynamic module of PR design while

realization of step 1 ,

2 and 4 makes up static implementation. For stereo audio processing, 4 filters per channel are designed for different cut off frequencies to provide user with selective band hearing facility. Instead

For video processing spatial filtering techniques is used for noise reduction by mean and median filters. The used architecture is based on an impulse response array of $5 \times 5$ mask. The idea of mean filtering is simply to replace each pixel value in an image with the mean value of its neighbors, including itself. This has the effect of eliminating pixel values, which are unrepresentative of their surroundings. Image averaging is a digital image processing technique (Oppenheim \& Schafer, 2000; Chanda et al., 2003) that is often employed to enhance video images that have been corrupted by random noise. The algorithm operates by computing an average or arithmetic mean of the intensity values for each pixel position in a set of captured images from the same scene or view field. The methodology of diffusing the intensity is

of having all the filters present at a time on device one filter per channel is loaded using PR and can be changed by user on the fly. Selection of filters was kept simple as the focus of work was to prove the usability of PR concept. Implementation of advanced filters using PR are expected to show same benefits as reconfigurable module is treated as black box as far as PR implementation is concerned.

System description

The implementation is done on ML-402 board consisting of Virtex-4 with support from VDEC1 card from Digilent. Video source is from NTSC/PAL compatible camera and the output display is $640 \times 480$ resolution VGA PC monitor. Audio source is from MP3 player and

Research article "Real time signal processing"

COIndian Society for Education and Environment (iSee) http://www.indjst.org
Sheetal et al. Indian J.Sci.Technol. 
the output device is headphone or speaker. The laboratory set up used for experimentation is shown in Fig. 2

Microblaze system is designed using Xilinx platform studio hardware implementation of audio, video is carried out in Xilinx ISE web pack and partial

Table 1. Resource utilization for implementations with and without $P R \&$ percentage saving in $P R$

\begin{tabular}{|l|l|l|l|}
\hline \multicolumn{3}{|c|}{ Resource utilization \& percentage saving } \\
\hline $\begin{array}{l}\text { Logic } \\
\text { utilization }\end{array}$ & $\begin{array}{l}\text { Used } \\
\text { without PR }\end{array}$ & $\begin{array}{l}\text { Used } \\
\text { With PR }\end{array}$ & $\begin{array}{l}\% \\
\text { saving }\end{array}$ \\
\hline $\begin{array}{l}\text { No. of slice flip } \\
\text { flops }\end{array}$ & 6086 & 3120 & 48.73 \\
\hline $\begin{array}{l}\text { No. of } 4 \text { input } \\
\text { LUTs }\end{array}$ & 9091 & 4166 & 54.17 \\
\hline
\end{tabular}

device Soft-core processor Microblaze is used from several available choices of processor cores like OpenRISC, LEON etc. Audio video processing

The filters for audio and video signals are implemented on hardware. Video decoder ADC ADV7183B is available on reconfiguration flow is carried out using early access plan-ahead tool (Jackson, 2007). Fig. 3 shows the block diagram of complete system. It consists of microblaze processor, hardware implementation of audio, video signals processing with partially reconfigurable filters. There are three reconfigurable modules, two audio filters for two audio channels and one video filter. The processor can reconfigure the audio and video filters as per user's choice by loading respective partial configuration bitstream through the ICAP port. The standalone operating system is running on microblaze allows managing the system, peripherals and PR. video decoder card which converts NTSC video signal into ITUR 656 format (Jack, 2005). This data is taken into FPGA and filtering is done as discussed in section III of this paper. Need of mean and median filter is mutually exclusive. Hence, PR is used to change averaging block by median calculating block to change the type of filter. Processed RGB signal is fed to Video DAC ADV7123 available on board; which converts it to VGA compatible signal. For audio signal LM 4550 audio codec is available on board. To control and configure it AC 97 core is generated and implemented in FPGA. Audio filters are also implemented using PR so as to have one filter per Table 2. Bit file sizes \& downloading time for implementations with \& without $P R$

\begin{tabular}{|l|l|l|l|l|}
\hline \multirow{3}{*}{ Implementation } & Type of bit file & $\begin{array}{l}\text { Size of } \\
\text { bit file }\end{array}$ & $\begin{array}{c}\text { Reconfig. time } \\
\text { Cable freq: 6MHz) } \\
\text { using JTAG }\end{array}$ & $\begin{array}{l}\text { Reconfig. time } \\
\text { using ICAP }\end{array}$ \\
\hline $\begin{array}{l}\text { Without PR } \\
\text { ALL Filters }\end{array}$ & Static bit file & $1673 \mathrm{~KB}$ & $6 \mathrm{sec}$ & - \\
\hline \multirow{5}{*}{ with PR } & Static bit file & $1673 \mathrm{~KB}$ & $6 \mathrm{sec}$ & - \\
\cline { 2 - 5 } & Partial mean filter & $190 \mathrm{kB}$ & $1 \mathrm{sec}$ & $360 \mathrm{mSec}$ \\
\cline { 2 - 5 } & Partial median filter & $244 \mathrm{~KB}$ & $1 \mathrm{sec}$ & $460 \mathrm{mSec}$ \\
\cline { 2 - 5 } & Video blank & $146 \mathrm{~KB}$ & $1 \mathrm{sec}$ & $280 \mathrm{mSec}$ \\
\cline { 2 - 5 } & Partial bit file left_LPF & $146 \mathrm{~KB}$ & $1 \mathrm{sec}$ & $280 \mathrm{mSec}$ \\
\cline { 2 - 5 } & Partial bit file_left_HPF & $135 \mathrm{~KB}$ & $1 \mathrm{sec}$ & $250 \mathrm{mSec}$ \\
\cline { 2 - 5 } & Partial bit file_left_BPF & $137 \mathrm{~KB}$ & $1 \mathrm{sec}$ & $260 \mathrm{mSec}$ \\
\cline { 2 - 5 } & Partial bit file_left_BSF & $130 \mathrm{~KB}$ & $1 \mathrm{sec}$ & $240 \mathrm{mSec}$ \\
\cline { 2 - 5 } & Partial bit file right_LPF & $135 \mathrm{~KB}$ & $1 \mathrm{sec}$ & $250 \mathrm{mSec}$ \\
\cline { 2 - 5 } & Partial bit file_right_HPF & $152 \mathrm{~KB}$ & $1 \mathrm{sec}$ & $290 \mathrm{mSec}$ \\
\cline { 2 - 5 } & Partial bit file_right_BPF & $138 \mathrm{~KB}$ & $1 \mathrm{sec}$ & $285 \mathrm{mSec}$ \\
\cline { 2 - 5 } & Partial bit file_right_BSF & $150 \mathrm{~KB}$ & $1 \mathrm{sec}$ & $108 \mathrm{mSec}$ \\
\cline { 2 - 5 } & Left_blank & $58 \mathrm{~KB}$ & $1 \mathrm{sec}$ & $\mathrm{mSec}$ \\
\cline { 2 - 5 } & Right_blank & $75 \mathrm{~KB}$ & $1 \mathrm{sec}$ & \\
\hline
\end{tabular}
channel available on FPGA.

Self reconfiguration \& user Interface

Self reconfiguration refers to handling on the fly PR by FPGA itself. Internal configuration access port (ICAP) is used for this (DS280, 2006). Partial bit files are stored in compact flash available on the board along with system ACE file. Microblaze drives the ICAP for changing the filters on the fly (Blodget, 2003). Decision of changing the filter can be taken by processor based on software running on it. For demonstration purpose we have used UART Hardware board

ML 402 (UG083, 2006) board has Virtex-4sx35ff668 (UG070, 2005) device which supports partial reconfiguration and has two ICAP cores on it. It has compact flash which is being used to store system ace and partial bit files. The UART port is used to provide user interface to select appropriate filter by user.

\section{Processor}

The choice of implementation for processor on FPGA is hard core or soft core processor. Soft-core processor provides benefits of easy implementation and upgradeability while hard core processors are best optimized hence provides higher Performance. As no hardcore processor is available on the

Table 3. Power dissipation for implementations with and without $P R$ and percentage saving in $P R$

\begin{tabular}{|l|l|l|l|}
\hline \multicolumn{4}{|c|}{ Power dissipation \& percentage saving } \\
\hline & $\begin{array}{c}\text { Used without } \\
\text { PR(W) }\end{array}$ & $\begin{array}{c}\text { Used with } \\
\text { PR(W) }\end{array}$ & $\begin{array}{c}\% \\
\text { saving }\end{array}$ \\
\hline Power & 8.65 & 7.65 & 11.56 \\
\hline
\end{tabular}

"Real time signal processing" http://www.indjst.org communication to show the menu and allow user to enter the choice of filter for audio and video signal processing. Though ICAP is used for reconfiguration FPGA can be partially or fully reconfigured using appropriate bit file using JTAG mode. This allows user to update hardware internally as well as externally if required (Xapp138, 2000).

Operating system

Selection of operating system is critical in any embedded system design. We have used standalone operating system provided by Xilinx as it is easy to use and good amount of documentation is available.

Experimental set up and result

- To validate and demonstrate

Sheetal et al. Indian J.Sci.Technol. 
the benefits of partial reconfiguration for early adaptation and resource utilization for computation intensive real time application in pervasive audio video filtering application is chosen as a bench mark circuit.

- To find out the benefit in terms of resource utilization; PR implementation, where only one filter for processing respective signals at a time is available in a system is compared with a system in which all filters are available all the time on the FPGA.

- To validate the early adaptation we have measured the reconfiguration time: time to partially reconfigure the FPGA through the ICAP. This time is compared with the time of total reconfiguration to adapt new filter. To observe the benefits of internal $\mathrm{PR}$, reconfiguration time is also compared with our earlier attempt of external PR; in which bit files were loaded with JTAG using Impact tool by Xilinx (Bhandari et al., 2009).

We also measured the third important parameter of any design i.e. power. Since the board hosting FPGA does not measurre power consumption, the power dissipation of the entire board is measured using the method of Paulsson et al, (2008). The clock frequency of ML 402 board is $100 \mathrm{MHz}$. Cable frequency for JTAG downloading is $6 \mathrm{MHz}$.

\section{Resource utilization}

Microblaze and other blocks of static module have a maximum area of 1587 slices. This leaves large space for hardware accelerator. The slices required for video filters are 206 and 668 for mean and median filters respectively. For audio filters required slices are 334 per filter per channel. The resource utilization for with and without PR implementation is presented in Table 1.

\section{Reconfiguration time}

In Table 2, sizes and downloading time of all static and partial bit files are loaded. After running through the partial reconfiguration, flow and assembling the $P R$ project static and partial bit files get generated. As only module needs to alter compared to the entire FPGA, the sizes of these partial bit files are smaller than static bit files. To download the bitstream we have used JTAG and ICAP ports. In the implementation where PR feature is not used we need to reconfigure entire FPGA which is done through JTAG. In the implementation where PR is used but not the ICAP bitstream downloading is done through ICAP. In the implementation where PR is done internally, ICAP is used. As in Table 2, the speed ups in adaptability are promising with the use of partial reconfiguration and ICAP port to handle it internally. This data allow determining that use of ICAP is justified. In Spartan 3 device, ICAP is not available. Recently, special core for such devices have been developed (Bayar \& Yurdakul, 2008).

Power dissipation and \% saving is shown in Table 3.

\section{Conclusion}

We have developed on the fly internal partial reconfiguration system for real time audio and video signal processing. The system consists of 3 independent reconfigurable modules with 2 and 4 choices for video and both audio filters. To handle reconfiguration internally, ICAP is used which is driven by software running on microbalze processor. Though audio and video filters are taken as case study, the observed benefits are independent of the application. Hence, the feature permits a virtually infinite number of coprocessor's configuration allowing wide performance for a wide amount of applications. Good amount saving in resource utilization is observed by using PR feature. For the applications where resources are not enough on the device can be fit by using PR. Use of PR feature also makes system easy and quick to adapt to the change in environment or as per need. Hence it is promising solution to the challenge raised by pervasive systems about easy configurability. Along with performance and utilization this feature proves itself beneficial for third critical dimension of design; i.e. power saving in PR system is also attractive benefit. Use of ICAP makes the system more autonomous in terms of changing the logic on the fly. It also reduces the reconfiguration time.

\section{References}

1. Bayar S and Yurdakul A (2008) Dynamic partial selfreconfiguration on spartan III FPGAs via a parallel configuration access port (PCAP). Proceedings of HiPEAC Workshop on Reconfigurable Computing, Goteborg, Sweden.

2. Bhandari S, Subbaraman S, Pujari S and Mahajan R (2009) Realtime video processing on FPFA using on the fly partial reconfiguration http://www.computer.org/ portal/web/csdl/ doi/10.1109/ICSPS.2009.32.

3. Blodget, B., McMillan, S., and Lysaght, P. 2003. A Lightweight Approach for Embedded Reconfiguration of FPGAs. In Proc. Conference on Design, Automation and Test in Europe - Volume 1 (March 03 - 07, 2003). Design, Automation, and Test in Europe. IEEE Computer Society, Washington, DC, 10399.

4. Chanda B and Majumdar D (2003) Digital image processing and analysis. $1^{\text {st }}$ edn. Prentice-Hall, India. pp103-109.

5. DS280 (2006) OPB HWICAP (v1.00.b) www.xilinx.com.

6. Jack K (2005) Video demystified: A handbook for digital engineer, 4th edn. Elsevier LLH Technology Publishing, Eagle Rock, VA. pp: 115-238.

7. Jackson B (2007) Partial reconfiguration design with Planahead 9.2 (v1.1) www.xilinx.com.

8. Koo C (2005) Benefits of partial reconfiguration. Xilinx Xcell J. $55,65-67$.

9. Lagger A (2006) Self reconfigurable platform for cryptographic application. In: Proc. Intl. Conf. on Field Programmable Logic \& Appln., Aug 28-30,Madrid, Spain.

10. Oppenheim AV and Schafer RW (2000) Discrete-time signal processing. $2^{\text {nd }}$ edn. Prentice-Hall, India. pp: 503-507.

11. Paulsson K, Hübner M, Bayar S and Becker J (2008) Exploitation of run-time partial reconfiguration for dynamic power management in Xilinx spartan III-based systems. In: Proc. Intl. Conf. on Field Programmable Logic \& Appln., (FPL 2008). Sept. 8-10. pp: 699-700.

12. UG070 (2005) Virtex-4 user guide. (v1.2) www.xilinx.com.

13. UG083 (2006) ML40X Getting started tutorial for evaluation platforms (v5.0). http://www.xilinx.com/ support/ documentation/boards_and_kits/ug083.pdf.

14. Xapp138 (2000) Virtex FPGA series configuration and readback (v2.0).

15. XAPP290 (2004) Two flows for partial reconfiguration: Module based and difference based (v1.2) http://www.xilinx.com/support/documentation/application_not es/xapp290.pdf. 